Conclusions: Preoperative administration of GCs decreases complications and LOS after major abdominal surgery as a likely consequence of attenuating the postsurgical inflammatory response. There is no evidence of increased complications in

\section{Abridged abstracts from the surgical} literature

\section{Preoperative Glucocorticoid Use in Major Abdominal Surgery: \\ Systematic Review and Meta-Analysis of Randomized Trials}

Sanket Srinivasa, MBChB, Arman A. Kahokehr, $\mathrm{MBChB}$, Tzu-Chieh Yu, MBChB, and

Andrew G. Hill, MD, FRACS, FACS

Ann Surg 2011;254:183191

Objective: To determine the clinical safety and efficacy of preoperative glucocorticoid (GC) administration in major abdominal surgery with regards to short term outcomes.

Background: Previous randomized controlled trials (RCTs) in major abdominal surgery have displayed conflicting results regarding the short-term benefits of preoperative GC. Importantly, the safety of this intervention has not been conclusively determined.

Methods: A systematic review and quantitative metaanalysis was conducted of all RCTs exploring preoperative $\mathrm{GC}$ administration in major abdominal surgery for the endpoints of complications, hospital length of stay (LOS) and serum IL-6 on postoperative day one. Subset analyses by procedure were planned "a priori."

Results: Eleven RCTs of moderate quality, comprising 439 patients in total, were included in the final analysis. Preoperative $\mathrm{GC}$ use decreased complications $(\mathrm{OR}=0.37 ; 95 \% \mathrm{CI}, 0.21-0.64 ; P<$ 0.01 ), $\operatorname{LOS}$ (mean $=1.97$ days; $95 \% \mathrm{CI},-3.33$ to -0.61 ; $P=0.01$ ), and serum IL-6 (mean: $55 \mathrm{pg} / \mathrm{mL} ; 95 \% \mathrm{CI}$, 82.30 to $-27.91 ; P<0.01)$. Preoperative GCs decreased complications in hepatic resection $(\mathrm{OR}=$ 0.28 ; 95\% CI, 0.14-0.55; $P<0.01)$ and mean LOS (mean LOS: -2.66 ; 95\% CI, -5.01 to $-0.32 ; P=0.03$ ). GCs reduced mean LOS in patients undergoing colorectal surgery (mean LOS: $-0.98 ; 95 \% \mathrm{CI},-1.67$ to $-0.27 ; P=0.01)$. There was no difference in complication rates (OR: $0.45 ; 95 \% \mathrm{CI}, 0.16-1.32 ; P=$ 0.15 ) or anastomotic leaks specifically. colorectal surgery.

\section{Abstractor's Comments}

This paper features a topic off the beaten track, of interest across surgical specialities. Fear of the impact of pharmacological doses of steroids on wound healing and on the susceptibility to infection often leads surgeons to balk at the prospect of perioperative steroid therapy. That said, many of the deleterious effects of the metabolic response to surgery are probably a consequence of the resultant acute inflammatory process and hence, it would not be counterintuitive to presume that the judicial use of steroids may be beneficial in this context. While inherent methodological constraints question the validity of the authors' conclusions here, the issue certainly warrants further investigation in view of its potential to reduce morbidity and related health costs. S Sivaganesh; MS, MRCS, PhD

\section{Familial colorectal cancer type $X$ : polyp burden and cancer risk stratification via a family history score}

Poh-Koon Koh, Matthew Kalady, Marek Skacel, Susan Fay, Ellen McGannon, Janet Shenal, Loretta Arroyo, Kathy Toderick and James Church.

ANZ J Surg 81(2011)

Background: Patients fulfilling Amsterdam-1 criteria without mismatch repair deficiency (termed familial colorectal cancer type X (FCC type X)) were reported to have lower cancer risks than classic Lynch syndrome. This study investigates the polyp and cancer burden of this population and demonstrates relationships with a family history score (FHS).

Methods: The Jagelman Registry was queried for patients meeting Amsterdam criteria with microsatellite stable/low colorectal cancers. The risk of colorectal neoplasia was ascertained using a published FHS. Polyp distribution, histology and cumulative counts as well as extra-colonic tumours in the pedigree were reviewed. 
Results: Twenty-one patients ( 9 males, 12 females) met study criteria. The median lifetime polyp count was 3 (range 136). FHS _ $8(80 \%)$ was significantly associated with an increased risk of colorectal cancer compared with those with scores $<8$ who are more likely to develop polyps $(P<\varangle .01)$. Twelve patients $(57 \%)$ had predominantly left-sided polyps. Ten colorectal cancers ( 7 left-sided, 3 right-sided) were diagnosed at a median age of 48 (range 3074) years. Only three tumours were mucinous or demonstrated tumour-infiltrating lymphocytes, typical of high microsatellite instability tumours. All patients had family history of colorectal cancers (CRCs) and at least 10 patients had a family history of uterine or breast cancer. One patient was found to have hyperplastic polyposis syndrome.

Conclusions: FCC type $\mathrm{X}$ likely represents a heterogenous group of as yet undefined CRC predispositions. The polyp burden and cancer risk are variable and can be somewhat delineated according to an FHS.

\section{Abstractor's Comments \\ About 20-30\% of colorectal cancers are familial. Well known are Lynch syndrome and Familial adenomatous polyposis(FAP). Lynch syndrome is caused by germline mutation in mismatch repair genes(MMR) resulting in abnormal repeat sequences(Microsatellite instability,MSI).}

About 50\% of patient fulfilling HNPCC criteria(Amsterdam or Bethesda) do not have MMR gene mutations. Lindor et al described this condition as 'Familial Colorectal Cancer Type $X$ '. These patients tends to have positive Amsterdam criteria and microsatellite stable(MSS) or Low(MSI-L) colorectal cancers

The study by Koh et al shows that that the polyps tend to be adenomatous or hyperplastic in FCC type X. Cancer are predominantly left sided. Family history score(FHS) is a useful tool to stratify the risk of both polyps and cancer. Intensive endoscopic surveillance is an effective risk reduction strategy. Neil Jayasuriya MBBS,MRCS, MS, FRACS
Therapeutic value and outcome of gastric access loops created during hepaticojejunostomy for iatrogenic bile duct injuries.

Jayasundara JA, de Silva WM, Pathirana AA

Surgeon. 2010 Dec;8(6):325-9. Epub 2010 Jun 30.

Background and Purpose: Hepaticojejunostomy is the reconstructive procedure performed for iatrogenic bile duct injuries. Anastomotic site stricture is the most significant complication of this operation. Revision surgery is associated with a significant morbidity and mortality. Creation of access to the anastomotic site facilitates the management of such strictures by minimal access techniques and reduces the need for revision surgery. This retrospective study aims to investigate the technical accessibility, usefulness, morbidity related to and the outcome of hepaticojejunostomy with gastric access loops performed as the treatment for iatrogenic bile duct injuries.

\section{Methodology:}

Twenty-seven consecutive patients who have undergone hepaticojejunostomy (including three revision surgeries and a re-revision surgery) with gastric access loops from July 2005 to October 2009 were followed up for clinical, biochemical, radiological and endoscopic evidence of anastomotic site occlusion and the need for intervention. Morbidity related to gastric access loops was assessed by dyspepsia disability score.

\section{Results:}

Mean follow up was 35.4 (range 6-61) months. Three patients developed anastomotic strictures at 4,22 and 5 months after hepaticojejunostomy and had successful endotherapy via the gastric access loop. They remain well at 33rd, 31st and 3rd months, respectively, following intervention. Based on the dyspepsia disability score none of the patients had symptomatic dyspepsia affecting daily activities.

\section{Conclusions:}

Gastric access loop is accessible and useful for stricture dilation and other endotherapeutic procedures. In the absence of significant symptoms related to bile reflux, gastric access loop could be considered as a useful and safe adjunct in the 
management of hepaticojejunostomy by surgeons especially in settings with limited facilities and expertise for radiological manipulations.

\section{Abstractor's Comments}

Traditionally surgeons have under taken the task of performing diagnostic and therapeutic endoscopy. The main drawback of hepatico-jejunostomy is the lack of endoscopic access to the biliary system for
Intervention. Construction of an additional gastric access loop seems to be a clever idea to overcome this issue. Few published trials with smaller number of cases have shown this to be a safe procedure. It will be interesting to evaluate the degree of biliary sepsis following gastric access loop in a larger cohort of patients as the traditional long Roux loops in excess of $40 \mathrm{~cm}$ is now bypassed by a shorter gastric access loop. Ruwan Wijesuriya; MS, MRCS 